\title{
Design a Fuzzy Logic Controller for a Rotary Flexible Joint Robotic Arm
}

\author{
Jamaludin Jalani*, and Suman Jayaraman \\ Department of Electrical Engineering Technology, Facuty of Engineering, University Tun Hussein Onn Malaysia, \\ Beg Berkunci 101, 86400, Parit Raja, Batu Pahat, Johor Darul Takzim
}

\begin{abstract}
The purpose of this research is to design a fuzzy logic feedback controller (FLC) in order to control a desired tip angle position a rotary flexible joint robotic arm. The FLC is also employed to dampen the vibration emanated from a rotary flexible joint robotic arm when reaching a desired tip angle position. The performance of FLC is tested in simulation and experiment. It is found that the FLC is successfully designed, applied and tested. The results show that fuzzy logic controller performed satisfactorily control a desired tip angle position and reduce the oscillations.
\end{abstract}

\section{Introduction}

Fuzzy Logic Control has been renowned control approach over the centuries. Fuzzy set theory was first introduced in a seminar paper by Lotfi Zadeh. It was published in the rather obscure journal of Information and Control [1]. Zadeh an electrical engineer by training was one of the leading authorities in control theory in the 1950 s and early 1960 s. However, during the process of writing a book on linear systems with Charles Desoer in 1963, he noticed that in spite of the richness of the existing mathematical theory of control, we have been able to deal with a very special case of systems that are linear and time invariant, or nonlinear but with a specific property [2]. He traced this problem back to the Aristotelian notion of absolute truth and falsehood, and generalized such notion to the case of partial truth and partial membership in a set.

By the late 1980's, with the advent of fuzzy chips, fuzzy logic was established successfully in Japan's industry. In contrast, the US contributed less application of fuzzy logic for their industry. This mainly due to the fact that, fuzzy logic lacked basic theoretical verifications concepts such as stability and robustness (i.e. mathematical model not available) which normally used by theoreticians for classical control. When a mathematical model is not available, it makes little sense to talk about stability or any other structural properties. Several researchers have tried to come up with stability conditions for fuzzy systems and have reported some success [3]. However, the primary goal of fuzzy logic is to develop an alternative to mathematical modeling for systems which either lack a proper mathematical model because it is either too ill-defined, or the model is so complicated that it is of no practical use [4].

The application of fuzzy logic began in 1974, when Mamdani and his associates used a fuzzy logic controller to control the temperature in a rotary cement kiln [5]. After that, more applications of fuzzy logic control were presented by researchers all over the world in various fields [6]-[12]. For example, the FLC has been used in pattern recognition, classification, and clustering and presented in [13]. Several applications for robotic have been reported in [4] and [5]. Moreover, [16] had applied the FLC to suppress the swing of gantry crane system. Meanwhile, application of fuzzy logic for renewable energy system has been presented by [17]. More application of FLC can be found in [18]-[20].

The application of FLC to control a rotary flexible joint module has been reported by several researchers. [21] had developed a composite collocated proportionalderivative (PD) and fuzzy logic control for tracking and vibration control. [22] had proposed a cascade fuzzy logic controller (FLC) to remove link vibrations and to obtain fast trajectory tracking performance. [23] had modelled and controlled the flexible joint for vertical orientation. Other control strategies for controlling a rotary flexible joint module can be found in [24], [25] and [26]. Although a fuzzy control approach has been used in their work, most of the control technique is complex and not straight forward. Hence a direct fuzzy control strategy is proposed for a rotary flexible joint in this paper. In particular, a fuzzy Mamdani is employed to overcome oscillations emanated from a rotary flexible joint robotic arm.

\section{Mathematical Equation}

In brief, Figure 1 depicts the flexible joint module coupled to the SRV02 plant. The Module is attached to the SRV02 load gear by two thumbscrews. The Main Arm is attached to the module body by two identical springs thus resulting in the flexible joint. In this paper, only the state space representation of the complete

\footnotetext{
* Corresponding author: jamalj@uthm.edu.my
} 
system is given. This allows us to investigate the fuzzy logic control performance in simulation. The detailed derivation of the mathematical equation of flexible joint module can be found in [15].

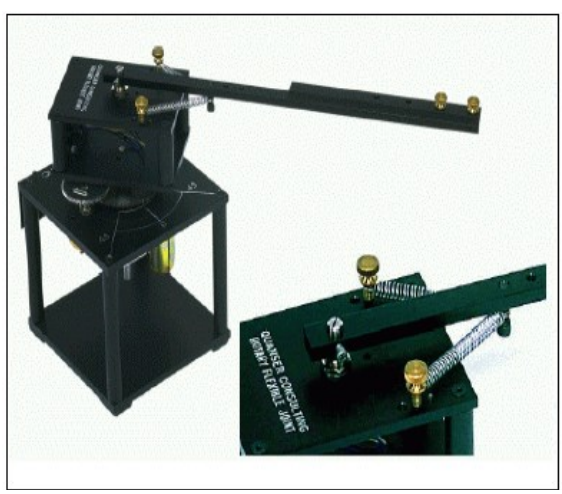

Fig. 1. Flexible Joint Module

$$
\begin{aligned}
& {\left[\begin{array}{c}
\dot{\theta} \\
\dot{\alpha} \\
\ddot{\theta} \\
\ddot{\alpha}
\end{array}\right]=\left[\begin{array}{cccc}
0 & 0 & 1 & 0 \\
0 & 0 & 0 & 1 \\
0 & \frac{K_{\text {stiff }}}{J_{e q}} & \frac{-\eta_{m} \eta_{g} K_{t} K_{m} K_{g}^{2}+B_{e q} R_{m}}{J_{e q} R_{m}} & 0 \\
0 & \frac{-K_{s t i f f}\left(J_{e q}+J_{A R M}\right)}{J_{e q} J_{A R M}} & \frac{\eta_{m} \eta_{g} K_{t} K_{m} K_{g}^{2}+B_{e q} R_{m}}{J_{e q} R_{m}} & 0
\end{array}\right]\left[\begin{array}{c}
\theta \\
\alpha \\
\dot{\theta} \\
\dot{\alpha}
\end{array}\right]} \\
& +\left[\begin{array}{c}
0 \\
\frac{\eta_{m} \eta_{g} K_{t} K_{g}}{J_{e q} R_{m}} \\
\frac{-\eta_{m} \eta_{g} K_{t} K_{g}}{J_{e q} R_{m}}
\end{array}\right] V_{1}
\end{aligned}
$$

\section{Experimental Set Up}

Similar experimental set up as in [24] is used for controlling the rotary flexible joint. In brief, $\mathrm{WinCon}^{\mathrm{TM}}$ is a real-time Windows $98 / \mathrm{NT} / 2000 / \mathrm{XP}$ application. It allows running code generated from a Simulink diagram in real-time on the same PC (also known as local PC) or on a remote PC. Data from the real-time running code may be plotted on-line in WinCon Scopes and model parameters may be changed on the fly through WinCon Control Panels as well as Simulink. The automatically generated real-time code constitutes a stand-alone controller (i.e. independent from Simulink) and can be saved in WinCon Projects together with its corresponding user-configured scopes and control panels.

\section{Fuzzy Logic Controller}

The goal of this project is to dampen oscillations of a rotary flexible joint robotic arm with fuzzy systems. The fuzzy logic controller is used because it is an innovative technology to design solutions for multiparameter and non-linear control problems. In addition, it uses human experience and experimental results rather than a mathematical model for the definition of a control strategy. Mathematical models and difference equations generate crisp descriptions of systems. This is fine if the mathematical properties and physical laws of the system are known or can be calculated. However, for non-linear processes, the underlying dynamics of the system can be too difficult, or indeed, impossible to model. In these situations, it is more useful to describe the system as a series of if-then rules. This is essentially what a fuzzy model is. It is a mapping of input space to output space by means of a rule base. Hence, the model requires no strict mathematical equations and the range of uses for the fuzzy model is vast. Figure 2 shows how fuzzy logic is used to control the position and to dampen the oscillation.

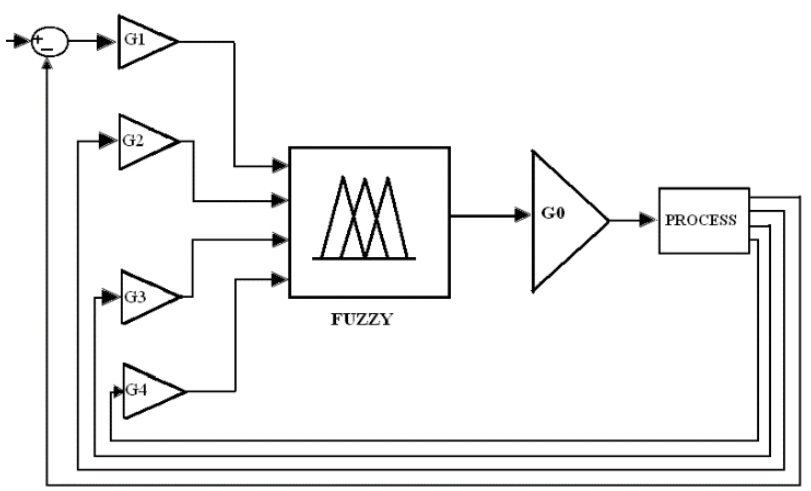

Fig. 2. The Fuzzy logic control block diagram for controlling flexible joint module

In general, the fuzzy controller has four components namely rule-base, interface mechanism, fuzzification interface and defuzzification interface as shown in Figure 3. A brief description of each component is as follows:

- The rule-base

Here, the knowledge for the control of the system is held as a set of IF - THEN statements.

- The inference mechanism

The inference mechanism evaluates which control rules are relevant at the current time and then decides what the input to the plant should be.

- Fuzzification interface

The fuzzification interface can be regarded as the input interface. It has the function of modifying/scaling the inputs so that they may compare to the rules in the rule base.

- Defuzzification interface

The defuzzification interface acts as an output interface. The output from the inference mechanism is converted so that it may be fed into the input for the process.

Figure 4 depicts the Rotary Flexible Joint controller with the fuzzy logic controller used for the experiment. Notice that both the actual system and an exact simulation are running in parallel thus to allow us comparing the actual and simulated results. This model has the I/O connection blocks linking to the physical plant as well as a simulated block to compare experimental and simulated results. 


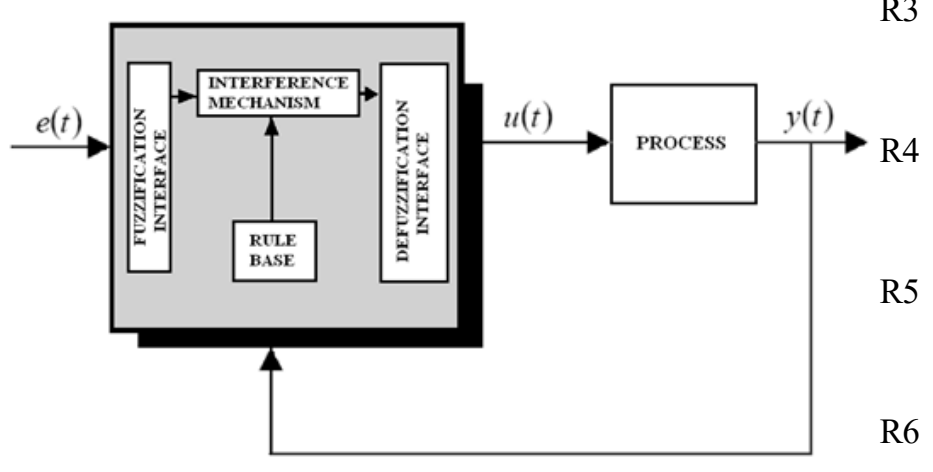

Fig. 3. Control system with fuzzy controller

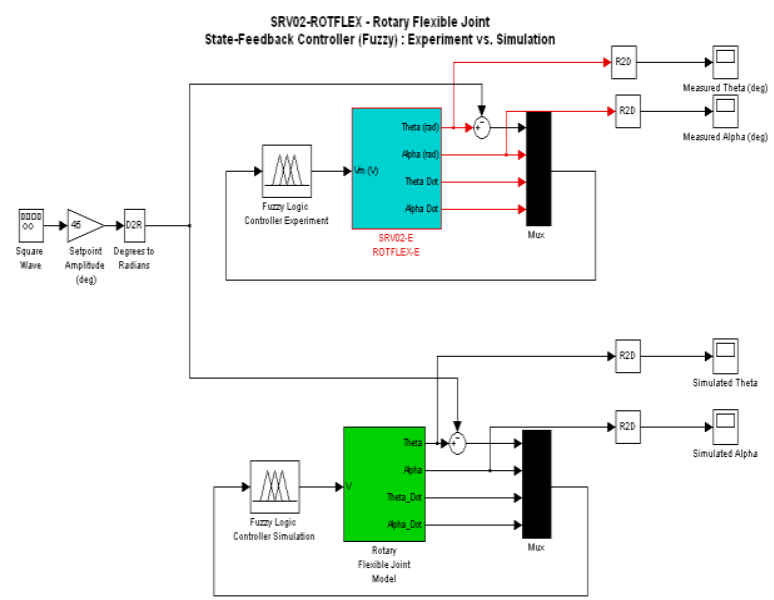

Fig. 4. Rotary Flexible Joint controller with the Fuzzy Logic controller.

\subsection{Fuzzy Mamdani Controller} 4.1.1 Input membership function

Theta Error (rad) $\rightarrow \quad$ neg-high, neg-low, zero,

Alpha (rad) $\quad \rightarrow \quad \begin{aligned} & \text { pos-low, pos-high. } \\ & \text { neg-high, neg-low, zero, }\end{aligned}$

pos-low, pos-high.

Theta Dot $\quad \rightarrow \quad$ neg, zero, pos.

Alpha Dot $\quad \rightarrow \quad$ neg, zero, pos.

\subsubsection{Output membership function}

Voltage (V) $\rightarrow$ neg, zero, pos

\subsubsection{Fuzzy Logic Rule-Base}

Fuzzy Logic Rule-Base applied for the rotary Flexible Joint Robotic Arm are as follows:

R1 : If (thetaerror is zero) and (alpha is zero) and (thetadot is zero) and (alphadot is zero) then (Voltage is zero)

R2 : If (thetaerror is neg-high) and (alpha is neghigh) and (thetadot is pos) and (alphadot is negative) then (Voltage is zero)
R3 : If (thetaerror is pos-high) and (alpha is poshigh) and (thetadot is neg) and (alphadot is positive) then (Voltage is zero)

If (thetaerror is neg-low) and (alpha is poshigh) and (thetadot is pos) and (alphadot is zero) then (Voltage is zero)

R5 : If (thetaerror is pos-low) and (alpha is neghigh) and (thetadot is neg) and (alphadot is zero) then (Voltage is zero)

R6 : If (thetaerror is neg-low) and (alpha is poslow) and (thetadot is zero) and (alphadot is positive) then (Voltage is pos)

R7 : If (thetaerror is pos-low) and (alpha is neglow) and (thetadot is zero) and (alphadot is negative) then (Voltage is neg)

R8 : If (thetaerror is neg-high) and (alpha is neghigh) and (thetadot is pos) and (alphadot is negative) then (Voltage is pos)

R9 : If (thetaerror is pos-high) and (alpha is poshigh) and (thetadot is neg) and (alphadot is positive) then (Voltage is neg)

R10 : If (thetaerror is zero) and (alpha is zero) and (thetadot is zero) and (alphadot is positive) then (Voltage is zero)

R11 : If (thetaerror is zero) and (alpha is zero) and (thetadot is zero) and (alphadot is negative) then (Voltage is zero)

R12 : If (thetaerror is zero) and (alpha is pos-high) and (thetadot is zero) and (alphadot is zero) then (Voltage is zero)

R13 : If (thetaerror is zero) and (alpha is neg-high) and (thetadot is zero) and (alphadot is zero) then (Voltage is zero)

R14 : If (thetaerror is zero) and (alpha is neg-low) and (thetadot is zero) and (alphadot is zero) then (Voltage is zero)

R15 : If (thetaerror is zero) and (alpha is pos-low) and (thetadot is zero) and (alphadot is zero) then (Voltage is zero)

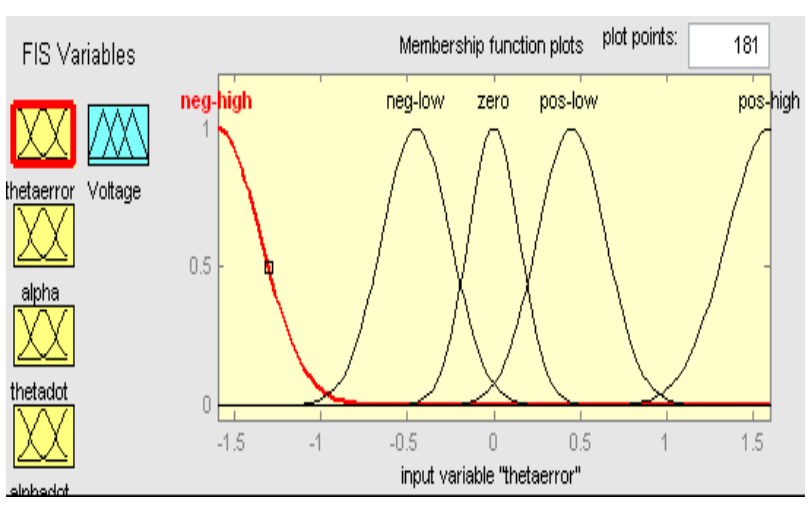

Fig. 5. FSMF for Theta Error. 


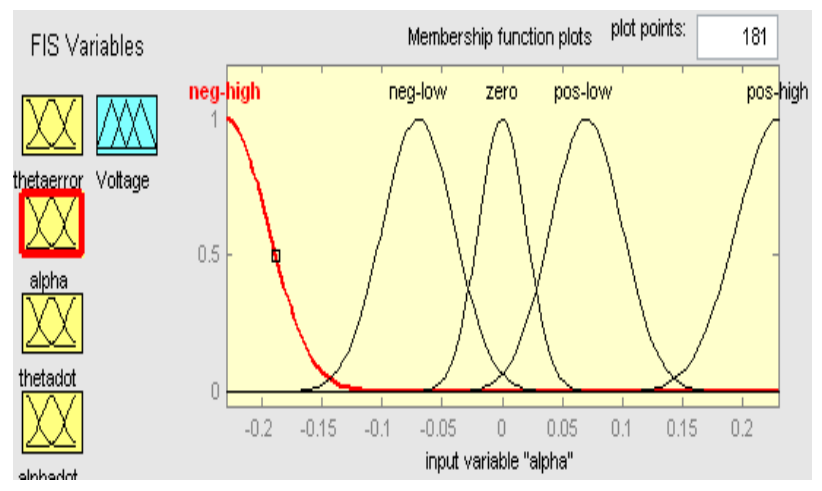

Fig. 6. FSMF for Alpha

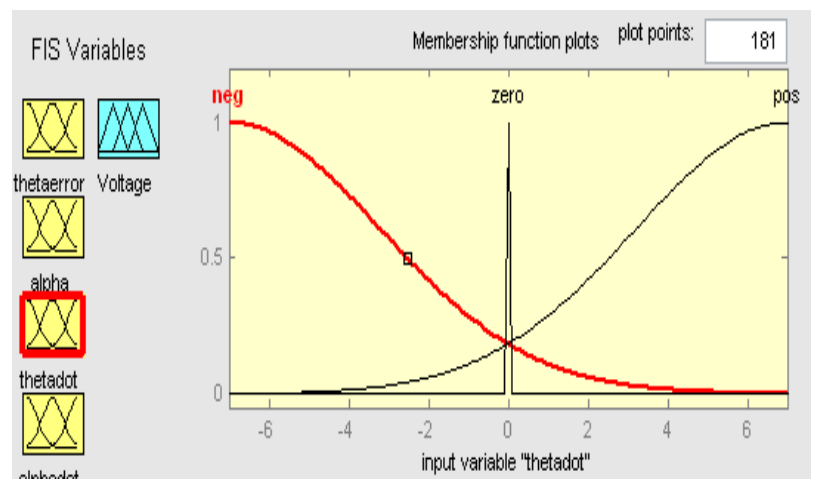

Fig. 7. FSMF for Theta Dot

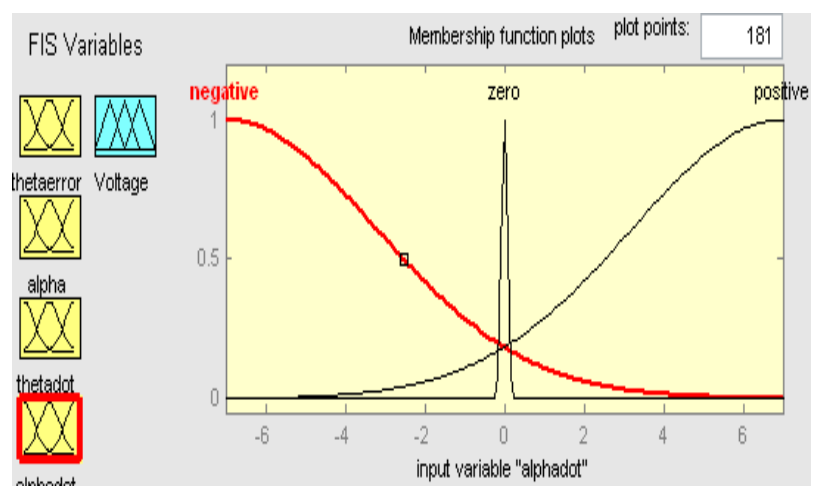

Fig. 8. FSMF for Alpha Dot

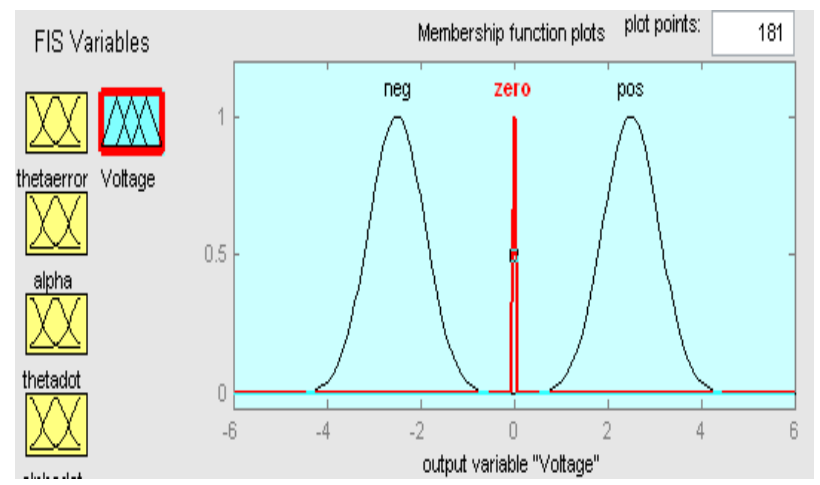

Fig. 9. FSMF for Voltage

The fuzzy set membership functions (FSMF) for the inputs are illustrated in Figure 5 to Figure 8. Meanwhile, the FSMF for the output illustrated in Figure 9.

\section{Results}

System performances by using Mamdani fuzzy controller is compared with the system without a controller in simulation and experiment. Simulated results are presented in Figure 10 and Figure 11. Meanwhile, experimental results are depicted in Figure 12 and Figure 13. The results show that the servo load angle did not achieve $45^{\circ}$. The maximum arm deflection angle is about $8^{\circ}$.

\subsection{Without Controller}

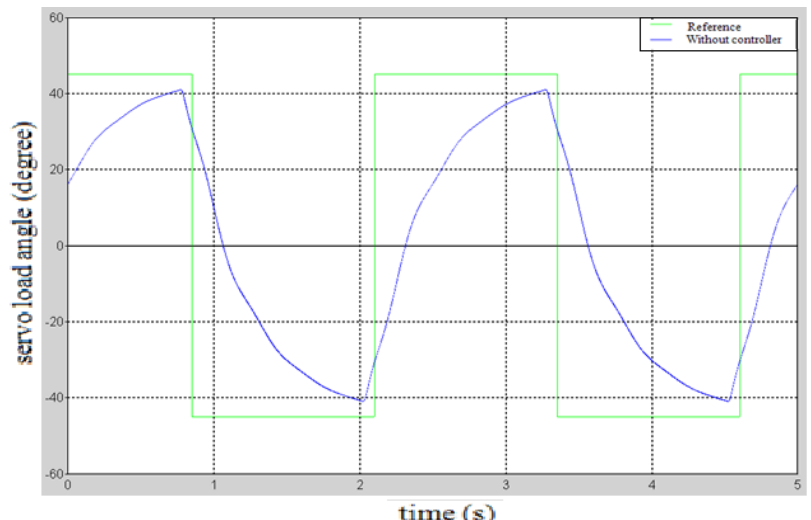

Fig. 10: Simulated plot for servo load angle without a controller.

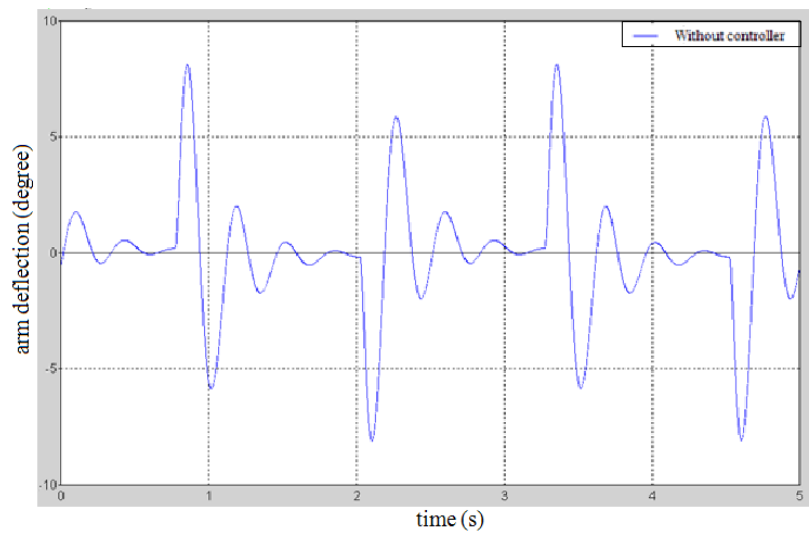

Fig. 11: Simulated plot for arm deflection angle without controller

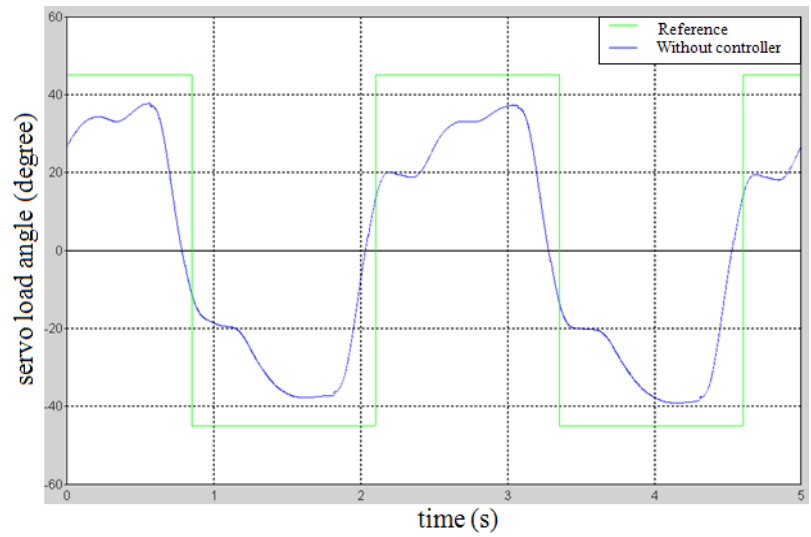

Fig. 12. Experimental plot for servo load angle without controller 


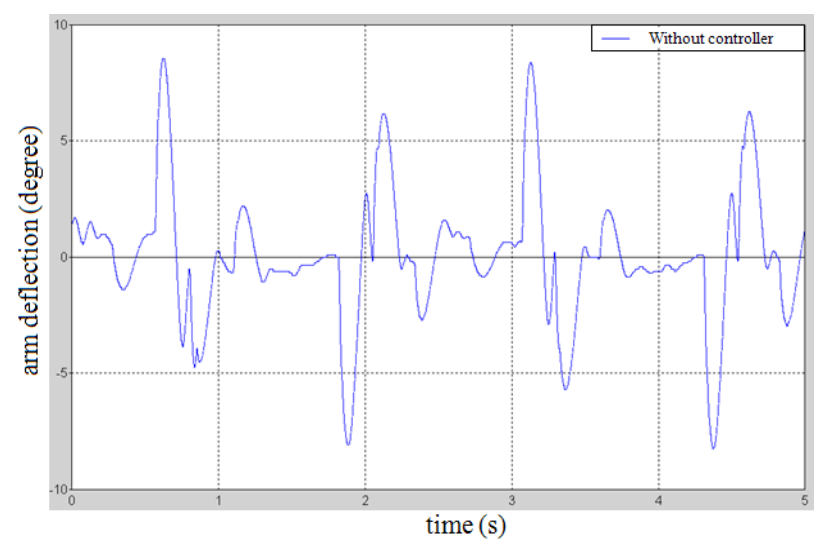

Fig. 13. Experimental plot for arm deflection angle without controller

\subsection{Simulation Results}

Simulated results for servo load angle and arm deflection angle with Mamdani Fuzzy controller is presented in Figure 14 and Figure 15.

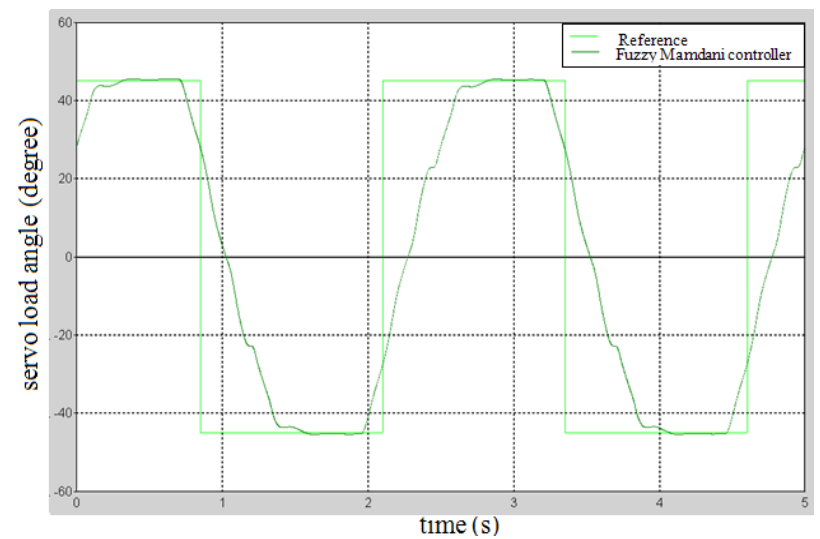

Fig. 14. Simulated plot for servo load angle with Fuzzy Mamdani controller

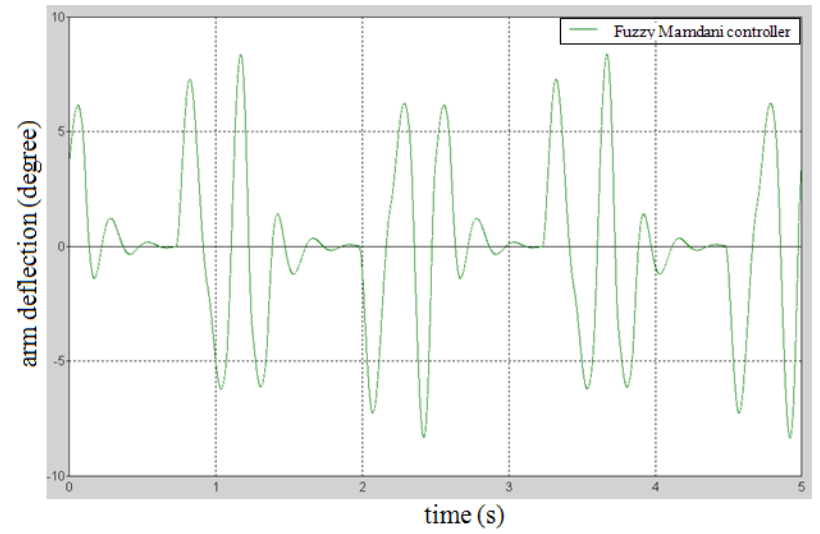

Fig. 15. The simulated plot for arm deflection angle with Fuzzy Mamdani controller.

\subsection{Experimental Results}

Experimental results for servo load angle and arm deflection angle with Fuzzy Mamdani controller is presented in Figure 16 and Figure 17.

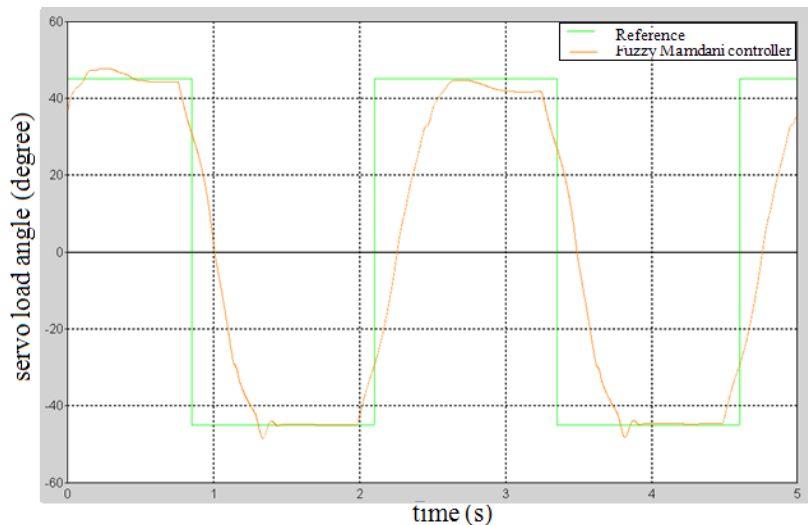

Fig. 16. Experimental plot for servo load angle with Fuzzy Mamdani controller

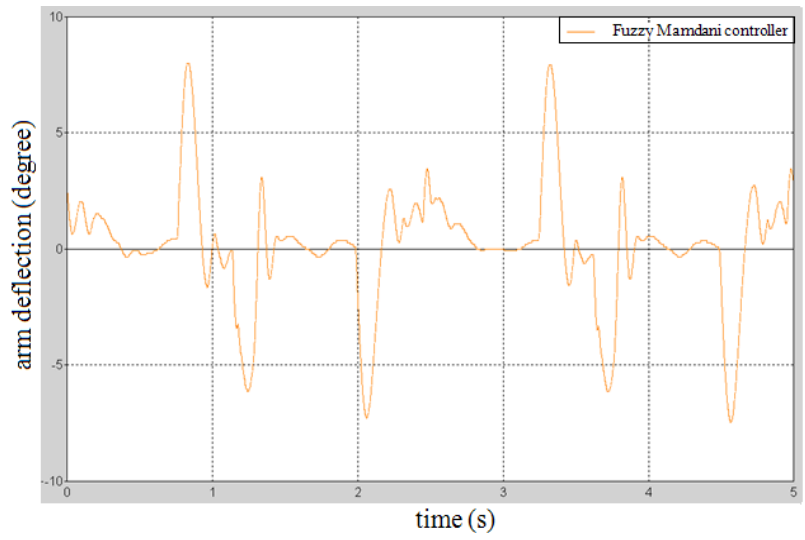

Fig. 17. The experimental plot for arm deflection angle with Fuzzy Mamdani controller.

A slight overshoot occurs in the experimental plot for servo load angle compared to the simulated plot. Meanwhile, the result of arm deflection angle results showed that the experimental plot is better than simulated plot. The arm deflection angle is about $8^{\circ}$.

\section{Conclusion}

The fuzzy logic controller was applied, designed and tested in the Rotary Flexible Joint Robotic Arm system. The Gaussian membership function was considered to develop the fuzzy rule. The results showed that fuzzy controller performed satisfactorily to suppress the oscillations. In future, the FLC should be further tuned so that the performances for servo load angle and arm deflection angle become better. The design may consider different membership function such as triangle and trapezoidal. The Fuzzy Sugeno may also be considered for future control design. 
The authors wish to thank the Faculty of Engineering Technology, Universiti Tun Hussein Onn Malaysia for providing a platform to carry out the research activities.

\section{References}

1 Zadeh and L. A, "Fuzzy Sets," Information and Control, vol. 8, no. 3, pp. 338-353, 1965.

2 L. A. Zadeh and C. A. Desoer, Linear System Theory: The State Space Approach. McGraw-Hill, 1963.

3 S. Thongchai and K. Kawamura, "Application of Fuzzy Control to a Sonar-Based Obstacle Avoidance Mobile Robot," Proceeding of the IEEE International Conference on Control Applications, pp. 425-430, 2000.

4 Pedro Albertos and Antonio Sala, "Fuzzy logic controllers Advantages and drawbacks," in IEEE Transactions On Control System, pp. 833-844.

5 E. H. Mamdani, "Application of Fuzzy Logic to Approximate Reasoning Using Linguistic Synthesis," IEEE Transaction Computer, vol. C-26, no. 12, pp. 17, 1977.

6 D. D. B. D. Rubin, G. Baselli, G. F. Inbar, and S. Cerutti, "An Adaptive Neuro-Fuzzy Method (ANFIS) for Estimating Single-Trial Movement-Related Potentials," Biological Cybernetics, vol. 75, pp. 63-75, 2004.

7 L. Birglen and C. M. Gosselin, "Fuzzy Enhanced Control of an Underactuated Finger Using Tactile and Position Sensors," Proceeding IEEE International Conference on Robotics and Automation, no. April, pp. 2320-2325, 2005.

8 J. Jantzen, "Design Of Fuzzy Controllers," Report Department of Automation, Technical University of Denmark, vol. 864, no. 98, pp. 1-27, 1998.

9 S. M. Hoseini, A. J. Koshkouei, and M. Farrokhi, "Chattering Free Fuzzy Sliding Mode Controllers For Robot Manipulators," Control, 2006.

10 F. Nagata, Y. Kusumoto, K. Hasebe, K. Saito, M. Fukumoto, and K. Watanabe, "Post Processor Using a Fuzzy Feed Rate Generator for Multi-Axis NC Machine Tools with a Rotary Unit," Proceeding of International Conference on Control, Automation and System, pp. 438-443, 2005.

11 J. Jalani, "Hardware-In-The-Loop Simulation (HILS) Based Design and Evaluation of an Intelligent Gantry Crane System," International Journal of Automation Technology, Vol. 1, No. 1, Pp. 11-19, 2007.

12 A. Bicchi, "Hands for Dexterous Manipulation And Robust Grasping: A Difficult Road Toward Simplicity," IEEE Transactions on Robotics and Automation, vol. 16, no. 6, pp. 652-662, 2000.

13 P. Melin and O. Castillo, "A Review On The Applications of Type-2 Fuzzy Logic In Classification And Pattern Recognition," Expert System Application, vol. 40, no. 13, pp. 5413-5423, 2013.

14 T. Doersam and O. Hammerschmidt, "An Adaptive Fuzzy Control for a Three Fingered Robot Gripper," Proceeding of Third Australian and New Zealand Conference on Intelligent Information Systems. ANZIIS, pp. 158-163, 1995.

15 L. C. Hun, J. Djuanda, W. E. Kiong, L. C. Ying, and T. C. Looi, "DSP-Based Fuzzy Logic Controller Using Memory Based Position Recognition For Robotic Arm
Manipulator," Proceeding of the $2^{\text {nd }}$ International Conference on Mechatronics, pp. 323-330, 2005.

16 J. Jalani, "Design and Implementation of Fuzzy Logic Controller for Intelligent Gantry Crane System," Proceeding of the $2^{\text {nd }}$ International Conference on Mechatronics, pp. 345-351, 2005.

17 L. Suganthi, S. Iniyan, and A. A. Samuel, "Applications of Fuzzy Logic In Renewable Energy Systems - A Review," Renewable and Sustainable Energy Reviews, vol. 48, pp. 585-607, 2015.

18 S. Lee, D. Kim, and G. Park, "Identification of Nonlinear Dynamic Systems via the Neuro-Fuzzy Computing and Genetic Algorithms," Proceeding of International Conference on Control, Automation and System, pp. 1892-1896, 2005.

19 V. V. K. Reddy and M. Sydulu, "Fuzzy and Genetic Approach for Optimal Capacitor Placement in Distribution Systems," 2006.

20 P. P. Bonissone, "Adaptive Neural Fuzzy Inference Systems (ANFIS): Analysis and Applications," pp. 1-41, 2002.

21 M. A. Ahmad, R. Mohd, T. Raja, M. S. Ramli, M. A. Zawawi, and M. H. Suid, "Composite PD and Fuzzy Logic Approach for Vibration of Elastic Joint Manipulator," International Conference on Control, Automation and Systems, 2010.

22 I. H. Akyuz, Z. Bingul, and S. Kizir, "Cascade Fuzzy Logic Control of A Single-Link FlexibleJoint Manipulator," Turkish Journal of Electrical Engineering and Computer Sicence, vol. 20, no. 5, pp. 713-726, 2012.

23 K. Groves and A. Serrani, "Modeling and Nonlinear Control of a Single-Link Flexible Joint Manipulator," no. 3, p. 13, 2010.

24 D. Sendrescu, "Nonlinear Identification of a Rotary Flexible Joint Experiment."

25 M. Rostami Kandroodi, M. Mansouri, M. Aliyari Shoorehdeli, and M. Teshnehlab, "Control of Flexible Joint Manipulator via Reduced RuleBased Fuzzy Control with Experimental Validation," ISRN Artificial Intelligence, vol. 2012, pp. 1-8, 2012.

26 R. A. Koshy and S. Thomas, "A Two-surface Discrete Sliding Mode Control based on Approach Angle Reaching Law," Proceeding of the International Multiconference of Engineers and Computer Scientists, vol. 2, 2012.

27 S. Yurkovich, S. Yurkovich, K. M. Passino, and K. M. Passino, "A Laboratory Course On Fuzzy Control," Educatiom IEEE Transaction, vol. 42, no. 1, pp. 15-21, 1999.

28 S. Yurkovich and K. M. Passino, Fuzzy Control. Addison Wesley Longman, 1998. 